\title{
Fecal microbiota and metabolome characteristics in cirrhotic patients accompanied with hepatic encephalopathy
}

xiao wei ( $\nabla$ xwei198672@163.com )

Institute of disease control and prevention

Jing Yuan

Centers for disease control and prevention

\section{Research article}

Keywords: cirrhosis, hepatic encephalopathy, gut, metabolome, intestinal microbiota

Posted Date: September 25th, 2019

DOI: https://doi.org/10.21203/rs.2.15183/v1

License: (c) (i) This work is licensed under a Creative Commons Attribution 4.0 International License. Read Full License 


\section{Abstract}

Background Gut microbiota is considered as an important endocrine organ and has remarkable influence on the metabolic phenotype of host, with extensive participation in the cometabolism with host. Significant changes occurred in intestinal microenvironment of cirrhotic patients under the influence of gut-liver axis. Hepatic encephalopathy is one of the common complications of cirrhosis. Methods In this study, four patients of cirrhosis accompanied with hepatic encephalopathy (CHE) were enrolled, with their healthy relatives as the controls, and paired comparison analysis was carried out. Fresh fecal samples were collected from participants, and subjected to Illumina MiSeq high throughput sequencing of 16S rRNA regions, and metabolome analysis was carried out using high-performance liquid phase chromatography and gas chromatography coupled with tandem mass spectrometry (HPLC-GC/MS-MS). Results Both the intestinal microbiota community and metabolome analysis showed distinct differences between $\mathrm{CHE}$ patients and the normal. CHE patients contained a remarkably decreased abundance of Bacteroidetes, whereas increased abundances of Firmicutes and Proteobacteria compared with the normal. Metabolome analysis revealed significant changes in metabolites constituents for CHE patients, showing distinct higher concentrations of amine, alanine, glutamic acid, ornithine, and tyrosine, with lower concentrations of cholesterol, myo-inositol, serine, suggesting the metabolic balance of intestinal microbiota was disrupted. The initial equilibrium state of gut microbiota community and metabolism was broken due to CHE. Conclusions Our study illustrated the intestinal microenvironment dysbiosis and dysfunction characteristics, which will be expected to provide guiding significance for intestinal microecological therapy and improvement of patients prognosis clinically.

\section{Background}

Gut microbiota has been neglected in precision medicine for a long time [1], and its importance has become increasingly evident with the advent of a large number of research, which continue to reveal the importance of intestinal microbiota in human health and disease [2, 3]. Gut microbiota is considered as an important endocrine organ, which can be adjusted or self-regulated [1]. Intestinal microbiota has intimate relationship with human disease and health, and has remarkable influence on the metabolic phenotype of host, with extensive participation in the cometabolism with host [3, 4]. The area of human gastrointestinal tract is achieved to $200-400 \mathrm{~m}^{2}$, and has important roles in promoting nutrient absorption, resisting infection, anti-aging, and promoting the development of the immune system [1]. Liver is the important metabolic organ in human body, and has multiple functions, sucn as detoxication, secretory protein synthesis, glycogen storage, and so on. Liver has intimate relationship with the gut through physiological structure of gut-liver axis [5]. Previous studies had suggested cirrhosis-specific characteristics of intestinal microbiota community and co-metabolome with the host[4, 6, 7]. Hepatic encephalopathy (HE) was the major complication for cirrhosis, and the liver was functional disordered in CHE patients, directly affecting the intestinal microenvironment, and further affecting gut microbiota community and metabolism.

In the normal human gut, amino acids from dietary intake are broken down by intestinal bacteria with the production of nitrogen-containing compounds, among which the most important nitrogenous waste products is ammonia $\left(\mathrm{NH}_{3}\right)$. The Ncontaining compounds are transported through the portal vein to the liver, where more than $80 \%$ are metabolised through the urea cycle pathway and/or excreted immediately. For cirrhotic patients, the liver cells are dysfunction and incapable of metabolising the waste products effectively. The redundant ammonia enter the blood circulation, cross the blood-brain barrier, and are further exploited by astrocyte to synthesise glutamine from glutamate. The surge in glutamine level causes brain edema, further increases the risk in developing hepatic encephalopathy [7]. It is reported that the risk of developing HE is up to $20 \%$ per year in cirrhotic patients [7].

In the past few years, more and more research were focused on the hepatopath related intestinal mcirobiota characteristics. Some clinical cases have shown that lowering serum ammonia levels with rifaximin, lactulose, probiotics and prebiotics can effectively reduce the severity of minimal hepatic encephalopathy (MHE), the principle of which is mainly to restore the normal state by adjusting the intestinal microenvironment, thereby reversing MHE [8]. This change further demonstrates the importance of intestinal microflora, ammonia, and inflammation in the process of MHE [8]. In 2016, fecal samples were collected from 40 controls and 147 cirrhotic patients ( 87 with $\mathrm{HE}$ ) to research on the relationship between fecal microbiota and brain MRI, with the results shown negative linkages between autochthonous families and positive ones between Enterobacteriaceae to MR

Page 2/15 
spectroscopy and hyperammonemia-associated astrocytic changes [9]. Bajaj JSet al. analyzed the gut microbial profile from twenty-five patients (17 HE and 8 without HE) and ten controls, which suggested that specific bacterial families (Alcaligeneceae, Porphyromonadaceae, Enterobacteriaceae) were strongly associated with cognition and inflammation in HE patients [10]. 2013, Tranah TH summarised the current evidence base which confirmed the synergistic role of systemic inflammation and hyperammonemia in the pathogenesis of HE [11]. In 2012, sixty cirrhotic patients (36 HE and 24 no-HE) and 17 controls were recruited and subjected to evaluation of fecal constituents, cognitive testing, cytokine and endotoxin analysis, which suggested that cirrhotic patients, especially HE patients, had significantly lower beneficial autochthonous and higher pathogenic genera compared with the normal, and HE patients had a lack of Roseburia and an overgrowth of Enterococcus, Veillonella, Megasphaera, and Burkholderia compared with no-HE patients [12]. 2010, Gupta A et al. confirmed high prevalence of small intestinal bacterial overgrowth (SIBO) in cirrhotic patients with minimal HE based on glucose breath test for SIBO and lactulose breath test from 102 cirrhotic patients (57 had HE) [13].

Given the above, the distribution of intestinal microbiota community can be learned by the changes of metabolites produced by intestinal microbiota. Alteration of metabolism in human gut synchronizes with the maturity and evolution of gut microbiota. Hepatopathy had a significant impact on the component and function of gut microbiota via gut-liver axis. However, few intensive studies have investigated the HE-related metabolic interactions between the gut and liver, as well as the correlation between gut microbiota component and metabolism. The gut microbiota community and co-metabolism had dramatic changes in CHE patients, which aroused our great interests. Our study highlighted the CHE specific gut microbiota community and metabolism, and researched how the intestinal microbiota regulated systemic metabolic changes in CHE patients.

\section{Methods}

\section{Study participants and fecal samples}

Hepatic encephalopathy was diagnosed by the confirmation of liver disease incorporated with encephalopathies. NCT-A (number connection test A) combined with DST (digit symbol test) were used in this study to define the presence of hepatic encephalopathy in cirrhotic patients. NCT-A combined with DST test was a widely accepted method to score hepatic encephalopathy among cirrhotic patients, meanwhile, this test had high reproducibility and diagnostic efficacy as validated by epidemiological survey[10]. The blood ammonia level in patients were detected using Blood ammonia analyzer (PA-4130). In this study, a total of 4 cirrhotic patients complicated with HE were recruited as case group, their healthy relatives were screened as the their controls, respectively. The paired comparison analysis was conducted. All healthy individuals had no evidence of hepatic or other diseases. In the process of enrolling, people who ingested probiotics, prebiotics, antibiotics, hormones, steroids within three monthes of sampling were excluded. The healthy family member of the patient was selected as the control because it took into account that the normal individual and patient have similar diets and living habits, which could avoid the influence of many confounding factors. In this study, we not only sample in pairs, but also pairwise comparison analysis to ensure more accurate analysis and more reliable results. This study was approved by the Institutional Review Board of Affiliated Hospital of Academy of Military Medical Sciences. All participants in this study signed an informed consent form prior to entering the study. This study conformed to the ethical guidelines of the 1975 Declaration of Helsinki.

\section{S rRNA gene sequencing}

Participants in this study were asked to provide a fresh stool sample, and metagenomic DNA were extracted using the QIAamp DNA stool Mini kit (Qiagen) following the manufacturer's instructions[14]. Metagenomic sequencing of 16S rRNA gene V1-V3 region was performed with the MiSeq platform (Illumina Inc., San Diego, CA) using version $3(300 \times 2)$ chemistry on the MiSeq instrument (Illumina) according to manufacturer's instructions [15]. Raw data preprocessing and analysis were performed using QIIME (version 1.9) [16]. UCHIME was used for low-quality reads filtering and chimeric sequences removing[17], and the highquality reads were clustered into operational taxonomic units (OTUs) with $97 \%$ of identity cutoff. Taxonomic affiliation of each OTU was assigned to the genus level against the Greengenes database[18].

\section{Metabolites extraction and identification}


Metabolites were extracted from fresh fecal samples taken from all subjects. A total of $1.2 \mathrm{ml}$ of cold $\left(-80^{\circ} \mathrm{C}\right)$ high performance liquid chromatography (HPLC)-grade methanol was added to the fecal samples, folloewed by vortex-mixing and sonicating for 30 seconds (Sonicator ${ }^{\circledR} 3000$; Misonix) on liquid nitrogen. This protocol was repeated 5 times, with storage at $-80^{\circ} \mathrm{C}$ for 60 min at the interval between each cycle. Samples were centrifuged at $1,3000 \mathrm{rpm}$ at $4^{\circ} \mathrm{C}$ for $20 \mathrm{~min}$ to remove the undissolved pellets, and the supernatant was collected and analysed by high-performance liquid phase chromatography and gas chromatography coupled with tandem mass spectrometry (HPLC-GC/MS-MS, Metabolon, Inc., North Carolina, USA) as described previously[19].

Samples were analyzed in one randomized run, during which time they were kept in the liquid phase chromatography autosampler at $4^{\circ} \mathrm{C}$. Molecular Feature Extraction (MFE) tool in the Mass Hunter Qualitative Analysis software (B.06.00, Agilent) was used to remove background noise and unrelated ions from the resulting data file. Mass Profiler Professional software (version 13.0, Agilent) was used to filter and align the primary data, which were then represented in a hierarchical condition tree (HCT). The identities were analysed by comparing the fragments obtained with the structure of the proposed compound in the MS/MS spectra provided in a public database (METLIN: https://metlin.scripps.edu/metabolites_list.php), as well as against commercially available standards. Wilcoxon rank-sum test method was used to assess the abundance of metabolites with significant differences between HE patients and the controls.

\section{Statistical analysis}

Microbiota community and metabolome data were repeated three times. Differential microbiota and metabolites between $\mathrm{CHE}$ patients and the normal were identified by Wilcoxon rank-sum test using SPSS for Windows (version 19.0 SPSS Inc., Chicago, IL, USA). The correlation analysis was performed by spearman rank correlation using R software. Statistic significance was denoted as $P<0.05$.

\section{Results}

\section{Methodology and study population}

In this study, we recruited and investigated cirrhotic patients accompanied with HE. Their healthy relatives were screened as the controls respectively. Paired comparison analysis was conducted in this study. The information and characteristics of all the recruited subjects were reported in Table 1. The normal human blood ammonia level (BAL) was $64.8 \pm 10.89$ umol/L. Abnormally high BALs were detected in the four CHE patients, with the average values were $71.8 \mathrm{umol} / \mathrm{L}, 92.7 \mathrm{umol} / \mathrm{L}, 104.2 \mathrm{umol} / \mathrm{L}, 105.5$ umol/L, respectively. A full description of the methods is available in the Materials and Methods section.

\section{Intestinal microbiota community in CHE patients}

The microbial community of fecal samples was analyzed by 16S rRNA gene sequencing using Illumina MiSeq instrument. The raw data of high-throughput sequencing has been submitted to NCBI (SRA accession: PRJNA534155). The HE patients carried less diverse gut microbiota compared with the normal. Bacterial abundance in each individual at the phylum, class, order, family, genus level was deposited in Table S1. The fecal microbiota structure could be clearly separated as revealed by PCA analysis (Fig. 1).. Heatmap analysis of bacteria abundance in each group was shown in Fig. 2. The differential bacteria were revealed by wilcoxon rank-sum test using SPSS for Windows and statistic significance was denoted as $P<0.05$. In general, at the phylum level, gut microbiota from patients contained higher abundance of Firmicutes, Proteobacteria, and lower abundance of Bacteroidetes. At the family level, the proportion of Veillonellaceae, Erysipelotrichaceae, Enterobacteriaceae, Streptococcaceae, and Pasteurellaceae were highly enriched in CHE patients. At the genus level, Veillonella, Streptococcus, Haemophilus, Lachnoclostridium, Megasphaera, Lactobacillus, Erysipelotrichaceae, Escherichia-Shigella had a higher level in CHE patients. Significantly decreased bacteria in CHE patients were mostly from Prevotellaceae and Acidaminococcaceae, and related genus including Ruminococcaceae, Phascolarctobacterium, Parasutterella, Subdoligranulum, Lachnospiraceae, Prevotella. 


\section{CHE specific changes in the predicted metabolites}

Metabolites constituents analysis was performed by HPLC-GC/MS-MS to explore global metabolic alterations associated with CHE. A total of $172,188,128,186$ metabolites were robustly identified in group 1 , group 2 , group 3 , group 4 , respectively. The differential metabolites were revealed by wilcoxon rank-sum test using SPSS for Windows and statistic significance was denoted as $P<0.05$. The abundance of metabolites in each group was compared separately, which exhibited distinct differences between CHE patients and the normal. Peak values of main metabolites detected from individuals in each group were exhibited in Fig. 3A. Metabolites constituents were significantly different between CHE patients and the normal as shown in Fig. 3B. In group 1, there were significantly enriched silanamine, butanediamine, hydroxylamine, butylamine, hexadecane, oleic acid, pentasiloxane, octane, ornithine, tyrosine, glutamic acid, sebacic acid, benzene, dioxolane, octadecadienoic acid, alanine, glycine were detected in CHE patient compared with the normal. In group 2, remarkably abundant of amine, hydroxylamine, methoxytryptamine, alanine, benzene, butane, dioxolane, glutamic acid, norvaline, octadecadienoic acid, oleic acid, ornithine, pentasiloxane, sebacic acid, tagatose, tyrosine were detected in CHE patient. In group 3, we detected significantly higher abundance of amine, alanine, butane, glycerol, glycine, hexadecane, octadecadienoic acid, octane, pentasiloxane, sebacic acid, tagatose in CHE patient. In group 4, CHE patient contained significantly higher level of alanine, benzene, dioxolane, glutamic acid, glycerol, glycine, hexadecane, norvaline, octane, oleic acid, ornithine, sebacic acid, tagatose, and tyrosine.

In general, seventeen metabolites were detected enriched, and seven metabolites were decreased in CHE patients. Abundance of differential metabolites in each individual were exhibited in Table S2. The multiple of difference of related metabolites was deposited in Fig. 4. The yellow background area represented the elevated metabolites in the patients, including alanine, benzene, butane, dioxolane, glutamic acid, glycerol, glycine, hexadecane, norvaline, octadecadienoic acid, octane, oleic acid, ornithine, pentasiloxane, sebacic acid, tagatose, tyrosine. The blue background area represented the reduced metabolites in the patients, including proline, cholesterol, myo-Inositol, acetic acid, serine, aspartic acid, valeric acid. The values represented the increased or decreased multiple of metabolites in CHE patients compared with the normal.

\section{CHE related correlations between microbiota and metabolites}

In this study, the correlation between differential mcirobiota community and metabolites abundance was analysed by spearman rank correlation and the heatmap was plotted using $\mathrm{R}$ software. As shown in Fig. 5, acetic acid, significantly decreased in CHE patients, had a strong and positive correlation with genera of Ruminococcaceae $(R=0.99)$ and Parasutterella $(R=0.99)$. Norvaline, which had a higher abundance in CHE patients, was positively correlated with Megasphaera $(\mathrm{R}=0.97)$, Veillonella $(\mathrm{R}$ $=0.86)$, Erysipelotrichaceae $(R=0.79)$, and Haemophilus $(R=0.74)$. Tagatose was more abundant in CHE patients, and its abundance was positively correlated with Streptococcus $(R=0.74)$, whereas negatively correlated with Phascolarctobacterium $(R=-0.70)$. Streptococcus was abundant in CHE patients and positively correlated with glycero $(R=0.78)$. Valeric acid showed positive correlations with genera of Parasutterella $(R=0.77)$, Ruminococcaceae $(R=0.72)$, and Erysipelotrichaceae $(R=0.62)$. In addition, positive correlations were detected between serine and Parasutterella $(R=0.70)$, octane and Prevotella $(R=0.66)$, as well as proline and Ruminococcaceae_NK4A214 ( $\mathrm{R}=0.66)$.

\section{Discussion}

Hepatic encephalopathy was regarded as a typical model of gut-liver-brain axis diseases [11]. Gut microbiota had extensive participation in human metabolism [4]. In cirrhotic patients, changes of intestinal microbial community and the metabolic byproducts, local and systemic inflammation, and intestinal barrier dysfunction will drive the development of hepatic encephalopathy [11].

In this study, we found that bacteria significantly increased in CHE patients were mostly from opportunistic pathogen, whereas the remarkably decreased bacteria were from autochthonous bacteria, suggesting the imbalance of intestinal microbiota community in CHE patients. Abnormally increased BALs were detected in CHE patients, accordingly, amino acid metabolism 
was enhanced and the abundance of ammonia was riched in fecal microbiota from CHE patients, which were presumed to be a potential factor to induce hepatic encephalopathy.

In addition, compared with their respective control, fecal samples from CHE patients contained a remarkable enrichment of alanine, glycine, norvaline, ornithine, and tyrosine. The concentration of norvaline was strongly correlated with genera of Megasphaera, Veillonella, Erysipelotrichaceae, and Haemophilus. Polar micelle was synthesized when bile salts in human gut aggregated into a certain concentration, followed by being carried to intestinal mucosa surface and absorbed [14]. Polymerized bile salts can be hydrolyzed by Veillonella, and the polar micelle formation was hindered, resulting in bile retention, biliary tract block, thus aggravating cirrhosis[14]. Haemophilus was the most notorious pathogen for human, and associated with various inflammatory diseases, such as bacteremia, meningitis, pneumonia, enteritis, and so on. Erysipelotrichaceae, belonging to phylum Firmicutes, were determined as opportunistic pathogens affecting various parts of human body, and related with metabolic disorders and intestinal infectious diseases [20]. Previous study had clearly suggested the positive correlations between Erysipelotrichaceae and metabolites from protein digestion[20].

Fecal samples from CHE patients were characterised by enhanced amino acids metabolism. Amino acids are catabolized mainly through urea cycle pathway, in which the amino group is removed, with nontoxic urea as the excreting formation, as well as uric acid or ammonia as the additional products[21]. In brief, the degradation of amino acids releases amines. In this study, some kinds of amine were detected enriched in CHE patients, including silanamine, butanediamine, hydroxylamine, butylamine, methoxytryptamine. In 2018, research from Wei mapped the cirrhosis-specific metabolic network of intestinal microbiota [22]. For cirrhotic patients, the liver is dysfunction and incapable of clearing away excessive ammonia and related nitrogenous substrates from the blood effectively, which can cross blood-cerebral barrier and are carried to brain, further affecting the normal operation of brain and facilitating the formation of hepatic encephalopathy [22]. The core of the metabolic network was the excessive production of amines, indicating that the abnormal metabolism of the intestinal microbiota and accumulation of amines were the potential risk of inducing hepatic encephalopathy. In this study, the characteristics of intestinal microbiota from CHE patients emphasized the enhanced amino acid metabolism and accumulation of amines, which was consistent with the research conclusion from Wei [22].

A remarkable increase in the abundance of glutamic acid was detected in CHE patients. Glutamic acid was synthesized through the pathway of aerobic fermentation of sugars and ammonia[22], with the predominant intestinal organism Corynebacterium glutamicum (also known as Brevibacterium flavum) being broadly used for production[23]. Glutamate was degraded into ammonium ion under the action of glutamate dehydrogenase in liver mitochondria, and further enter urea cycle to produce urea.

Tyrosine was detected in significantly higher amount in CHE patients. Tyrosine could be metabolised by intestinal decarboxylase with the production of tyramine, which will form octopamine under the action of $\beta$-hydraxylase, following by entering the nervous system [11]. Octopamine was in high concentration in CHE patients because of incomplete removal by liver, in addition, octopamine had the similar chemical construction with norepinephrine and replaced normal neurotransmitter, arising abnormal inhibition, consciousness disorder, and coma, and so on [11].

A higher abundance of ornithine was detected in fecal samples from CHE patients. Ornithine, as the substrate of the internal ornithine cycle, almost involved in the whole process of urea cycle and ammonia detoxification [11]. Liver was dysfunctional and urea cycle was impaired in CHE patients, resulting in excessive ammonia produced by intestinal microbiota which couldn't be cleaned away effectively, as a compensatory mechanism, enriched ornithine was produced in intestine to transform the toxic ammonia to urea.

Cholesterol showed a remarkable number of decrease in CHE patients. Cholesterol originates from the diet, bile, as well as desquamated intestinal cells, and can be metabolized by the intestinal microbiota. Cholesterol is oxidized by the liver to produce multiple bile acids [24], which are in turn conjugated with glycine, taurine, glucuronic acid, or sulfate. Cholesterol is excreted into the bile from the liver, along with a mixture of conjugated and nonconjugated bile acids. Approximate $95 \%$ of the bile acids are reabsorbed in the intestines, and the remainder are excreted in the feces. CHE patients had decreased serum cholesterol level, the same was true of cholesterol level in fecal metabolites.

Page 6/15 


\section{Conclusions}

Gut microbiota was regarded as an organ which can be adjusted or self-regulated, confirming that the correlation between gut microbiota and the human body is not just coexistence, but mutually beneficial relationships. Our data demonstrated that the abnormal metabolism of the intestinal microbiota leads to excessive production of amines in CHE patients. The liver function is metabolically incapable and can't effectively eliminating the amines in the body, leading to the accumulation of amines, which is considered to be an important factor in cirrhotic patients suffering from hepatic encephalopathy. Data in this study will provide theoretical guidance for active regulating the balance of structure and metabolism of intestinal microbiota, in order to further prevent the deterioration and improve the prognosis of the disease clinically in the future.

\section{Abbreviations}

CHE, Cirrhosis accompanied with hepatic encephalopathy

HCT, hierarchical condition tree

HPLC-GC/MS-MS, high-performance liquid phase chromatography and gas chromatography coupled with tandem mass spectrometry

MFE, Molecular Feature Extraction

OTU, operational taxonomic unit

SIBO, small intestinal bacterial overgrowth

\section{Declarations}

\section{Ethics approval and consent to participate}

This study was approved by the Institutional Review Board of Affiliated Hospital of Academy of Military Medical Sciences. All participants signed an informed consent form prior to entering the study. The study conformed to the ethical guidelines of the 1975 Declaration of Helsinki.

\section{Consent to publish}

All authors approve to publish this paper on BMC gastroenterology.

We have obtained verbal consent to publish from the participants to report individual data.

Availability of data and materials

All data generated or analysed during this study are included in this published article and its supplementary information files.

Competing interests

None to declare.

Funding

No funding. Not applicable.

\section{Authors' Contributions}


XW designed research, performed research, and contributed reagents or analytic tools; JY analyzed data and wrote the paper. All authors have read and approved the manuscript.

Acknowledgements

Not applicable.

\section{Authors' Information}

\section{Xiao Wei, Email: xwei198672@163.com}

\section{Jing Yuan, Email: yuanjing6216@163.com}

\section{References}

1.Clarke G, Stilling RM, Kennedy PJ, Stanton C, Cryan JF, Dinan TG: Minireview: Gut microbiota: the neglected endocrine organ. Molecular endocrinology 2014, 28(8):1221-1238.

2.Qin J, Li R, Raes J, Arumugam M, Burgdorf KS, Manichanh C, Nielsen T, Pons N, Levenez F, Yamada T et al: A human gut microbial gene catalogue established by metagenomic sequencing. Nature 2010, 464(7285):59-65.

3.Qin J, Li Y, Cai Z, Li S, Zhu J, Zhang F, Liang S, Zhang W, Guan Y, Shen D et al: A metagenome-wide association study of gut microbiota in type 2 diabetes. Nature 2012, 490(7418):55-60.

4.Wei X, Jiang S, Zhao X, Li H, Lin W, Li B, Lu J, Sun Y, Yuan J: Community-Metabolome Correlations of Gut Microbiota from Child-Turcotte-Pugh of $A$ and B Patients. Frontiers in microbiology 2016, 7:1856.

5.Szabo G: Gut-liver axis in alcoholic liver disease. Gastroenterology 2015, 148(1):30-36.

6.Yamashiro Y: Gut Microbiota in Health and Disease. Annals of nutrition \& metabolism 2017, 71(3-4):242-246.

7.Bajaj JS: Review article: the modern management of hepatic encephalopathy. Alimentary pharmacology \& therapeutics 2010, 31(5):537-547.

8.Felipo V, Urios A, Montesinos E, Molina I, Garcia-Torres ML, Civera M, Olmo JA, Ortega J, Martinez-Valls J, Serra MA et al: Contribution of hyperammonemia and inflammatory factors to cognitive impairment in minimal hepatic encephalopathy. Metabolic brain disease 2012, 27(1):51-58.

9.Ahluwalia V, Betrapally NS, Hylemon PB, White MB, Gillevet PM, Unser AB, Fagan A, Daita K, Heuman DM, Zhou H et al: Impaired Gut-Liver-Brain Axis in Patients with Cirrhosis. Scientific reports 2016, 6.26800.

10.Bajaj JS, Ridlon JM, Hylemon PB, Thacker LR, Heuman DM, Smith S, Sikaroodi M, Gillevet PM: Linkage of gut microbiome with cognition in hepatic encephalopathy. American journal of physiology Gastrointestinal and liver physiology 2012, 302(1):G168-175.

11.Tranah TH, Vijay GK, Ryan JM, Shawcross DL: Systemic inflammation and ammonia in hepatic encephalopathy. Metabolic brain disease 2013, 28(1):1-5.

12.Bajaj JS, Hylemon PB, Ridlon JM, Heuman DM, Daita K, White MB, Monteith P, Noble NA, Sikaroodi M, Gillevet PM: Colonic mucosal microbiome differs from stool microbiome in cirrhosis and hepatic encephalopathy and is linked to cognition and inflammation. American journal of physiology Gastrointestinal and liver physiology 2012, 303(6):G675-685. 
13.Gupta A, Dhiman RK, Kumari S, Rana S, Agarwal R, Duseja A, Chawla Y: Role of small intestinal bacterial overgrowth and delayed gastrointestinal transit time in cirrhotic patients with minimal hepatic encephalopathy. Journal of hepatology 2010, 53(5):849-855.

14.Wei X, Yan X, Zou D, Yang Z, Wang X, Liu W, Wang S, Li X, Han J, Huang L et al: Abnormal fecal microbiota community and functions in patients with hepatitis B liver cirrhosis as revealed by a metagenomic approach. BMC gastroenterology 2013, 13:175.

15.Kozich JJ, Westcott SL, Baxter NT, Highlander SK, Schloss PD: Development of a dual-index sequencing strategy and curation pipeline for analyzing amplicon sequence data on the MiSeq Illumina sequencing platform. Applied and environmental microbiology 2013, 79(17):5112-5120.

16.Caporaso JG, Kuczynski J, Stombaugh J, Bittinger K, Bushman FD, Costello EK, Fierer N, Pena AG, Goodrich JK, Gordon JI et al: QIIME allows analysis of high-throughput community sequencing data. Nature methods 2010, 7(5):335-336.

17.Edgar RC: UPARSE: highly accurate OTU sequences from microbial amplicon reads. Nature methods 2013, 10(10):996-998.

18.McDonald D, Price MN, Goodrich J, Nawrocki EP, DeSantis TZ, Probst A, Andersen GL, Knight R, Hugenholtz P: An improved Greengenes taxonomy with explicit ranks for ecological and evolutionary analyses of bacteria and archaea. The ISME journal 2012, 6(3):610-618.

19.Vernocchi P, Del Chierico F, Putignani L: Gut Microbiota Profiling: Metabolomics Based Approach to Unravel Compounds Affecting Human Health. Frontiers in microbiology 2016, 7:1144.

20.Bermingham EN, Maclean P, Thomas DG, Cave NJ, Young W: Key bacterial families (Clostridiaceae, Erysipelotrichaceae and Bacteroidaceae) are related to the digestion of protein and energy in dogs. PeerJ 2017, 5:e3019.

21.Weissenborn K: Minimal hepatic encephalopathy: a permanent source of discussion. Hepatology 2002, 35(2):494-496.

22. Wei X, Zhao J, Jia X, Zhao X, Li H, Lin W, Feng R, Yuan J: Abnormal Gut Microbiota Metabolism Specific for Liver Cirrhosis. Frontiers in microbiology 2018, 9:3051.

23.Foley P, Kermanshahi pour A, Beach ES, Zimmerman JB: Derivation and synthesis of renewable surfactants. Chemical Society reviews 2012, 41(4):1499-1518.

24.Long SL, Gahan CGM, Joyce SA: Interactions between gut bacteria and bile in health and disease. Molecular aspects of medicine 2017, 56:54-65.

\section{Tables}

Table 1. Clinical characteristics of subjects in this study. 


\begin{tabular}{|c|c|c|c|c|c|c|c|c|}
\hline \multirow[t]{2}{*}{ Characteristics } & \multicolumn{2}{|l|}{ Group 1} & \multicolumn{2}{|l|}{ Group 2} & \multicolumn{2}{|l|}{ Group 3} & \multicolumn{2}{|l|}{ Group 4} \\
\hline & HE1 & NM1 & HE2 & NM2 & HE3 & NM3 & HE4 & NM4 \\
\hline Age & 52 & 53 & 59 & 57 & 41 & 42 & 42 & 44 \\
\hline $\begin{array}{l}\text { BMIindex } \\
\left(\mathrm{kg} / \mathrm{m}^{2}\right)\end{array}$ & 27.71 & 26.9 & 28.4 & 26.61 & 29.86 & 27.73 & 28.14 & 24.36 \\
\hline Ascites & None & - & None & - & None & - & None & - \\
\hline $\begin{array}{l}\text { Prothrombin } \\
\text { time (seconds) }\end{array}$ & 14.9 & - & 12.8 & - & 14.9 & - & 16.8 & - \\
\hline Albumin (g/dL) & 29 & - & 24 & - & 28 & - & 31 & - \\
\hline $\begin{array}{l}\text { Total bilirubin } \\
(\mu \mathrm{mol} / \mathrm{L})\end{array}$ & 31.2 & - & 35.7 & - & 55.1 & - & 39.6 & - \\
\hline $\begin{array}{l}\text { Blood } \\
\text { ammonia } \\
\text { (umol/L) }\end{array}$ & $71.8 \pm 16.9$ & - & $92.7 \pm 21.1$ & - & $104.2 \pm 25.3$ & - & $105.5 \pm 31.9$ & - \\
\hline Complication & $\begin{array}{c}\text { hepatic } \\
\text { encephalopathy }\end{array}$ & - & $\begin{array}{c}\text { hepatic } \\
\text { encephalopathy }\end{array}$ & - & $\begin{array}{c}\text { hepatic } \\
\text { encephalopathy }\end{array}$ & - & $\begin{array}{c}\text { hepatic } \\
\text { encephalopathy }\end{array}$ & - \\
\hline $\begin{array}{l}\text { Child-Pugh } \\
\text { score }\end{array}$ & 6 & - & 8 & - & 9 & - & 7 & - \\
\hline
\end{tabular}

\section{Figures}




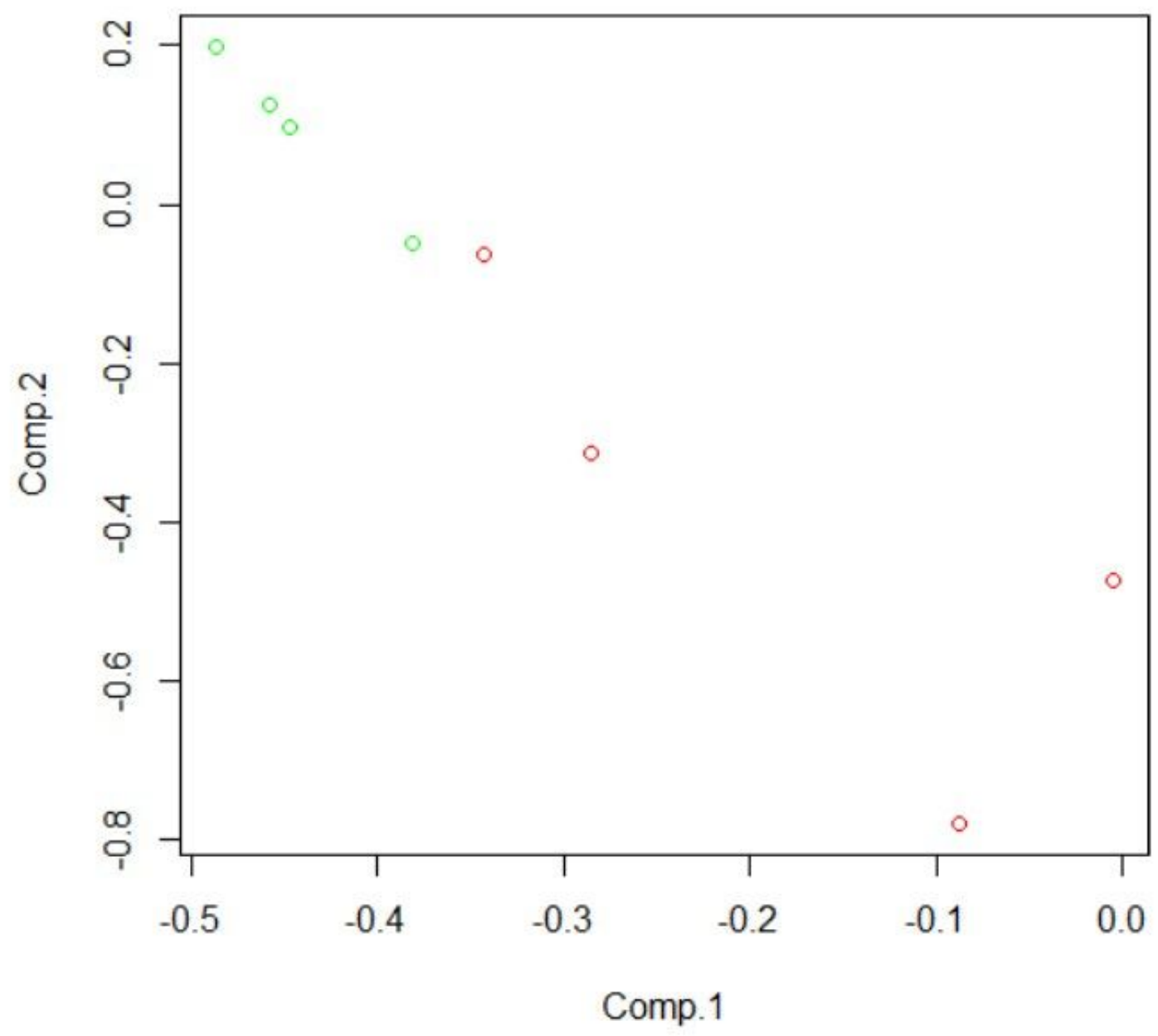

\section{Figure 1}

PCA analysis demonstrated the significant difference of fecal microbiota community between HE patients and the normal. Green circle represents the normal, red circle represents the HE patients. 


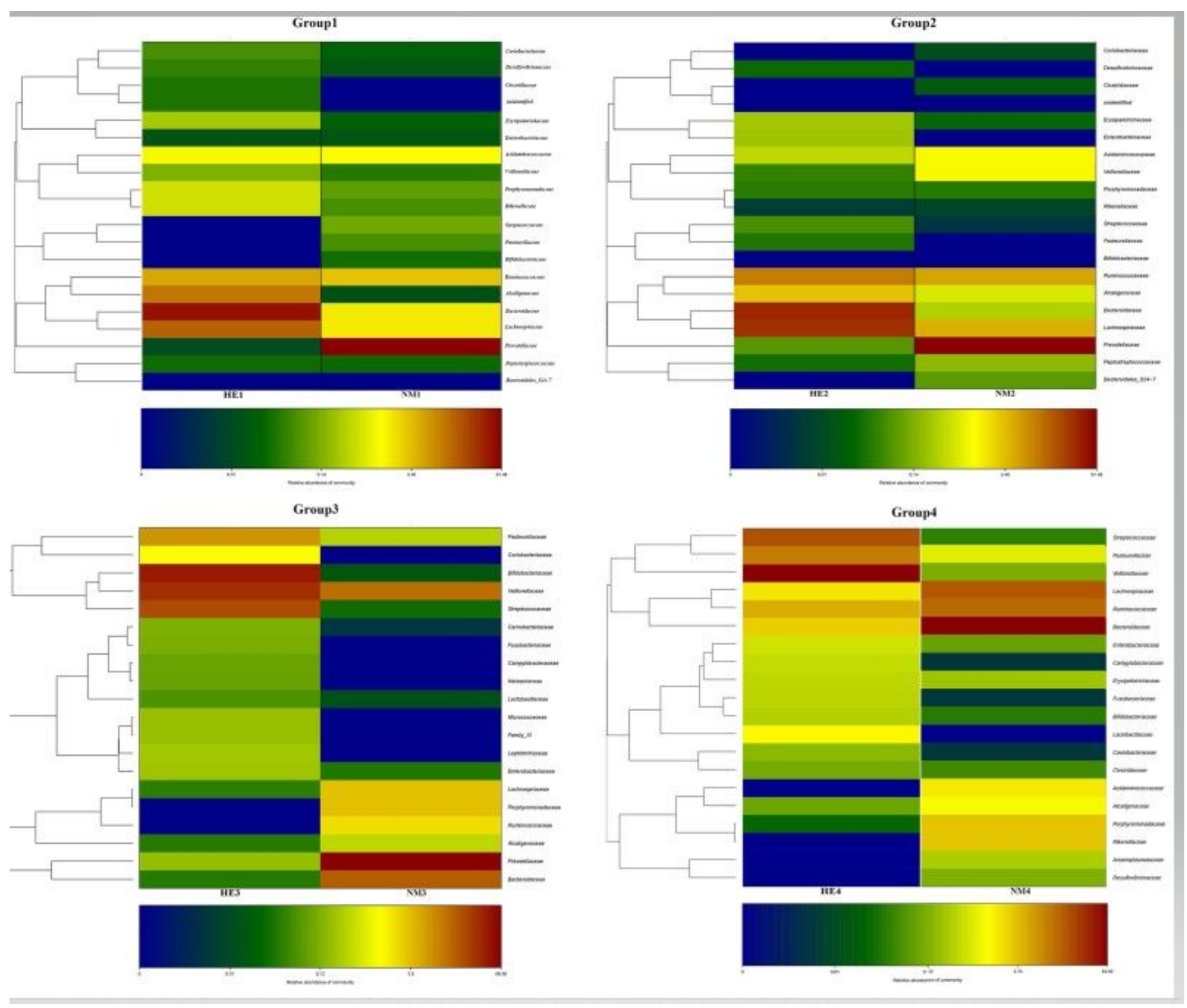

Figure 2

Heat-map analysis of intestinal microbiota community at the family level in each group. The color scale represented the scaled abundance of the bacteria at the family level, denoted as Z-score, with red and blue indicating increased and decreased abundances, respectively. 
A
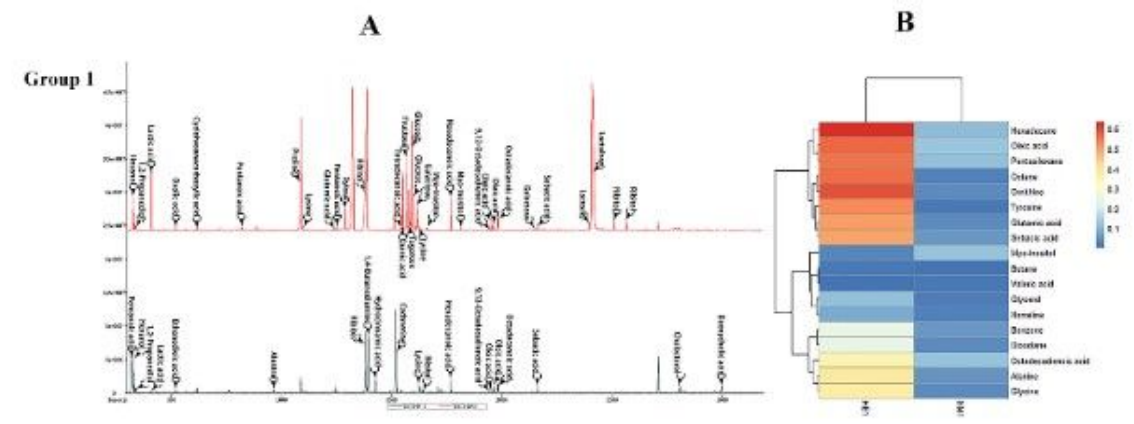

Group 2
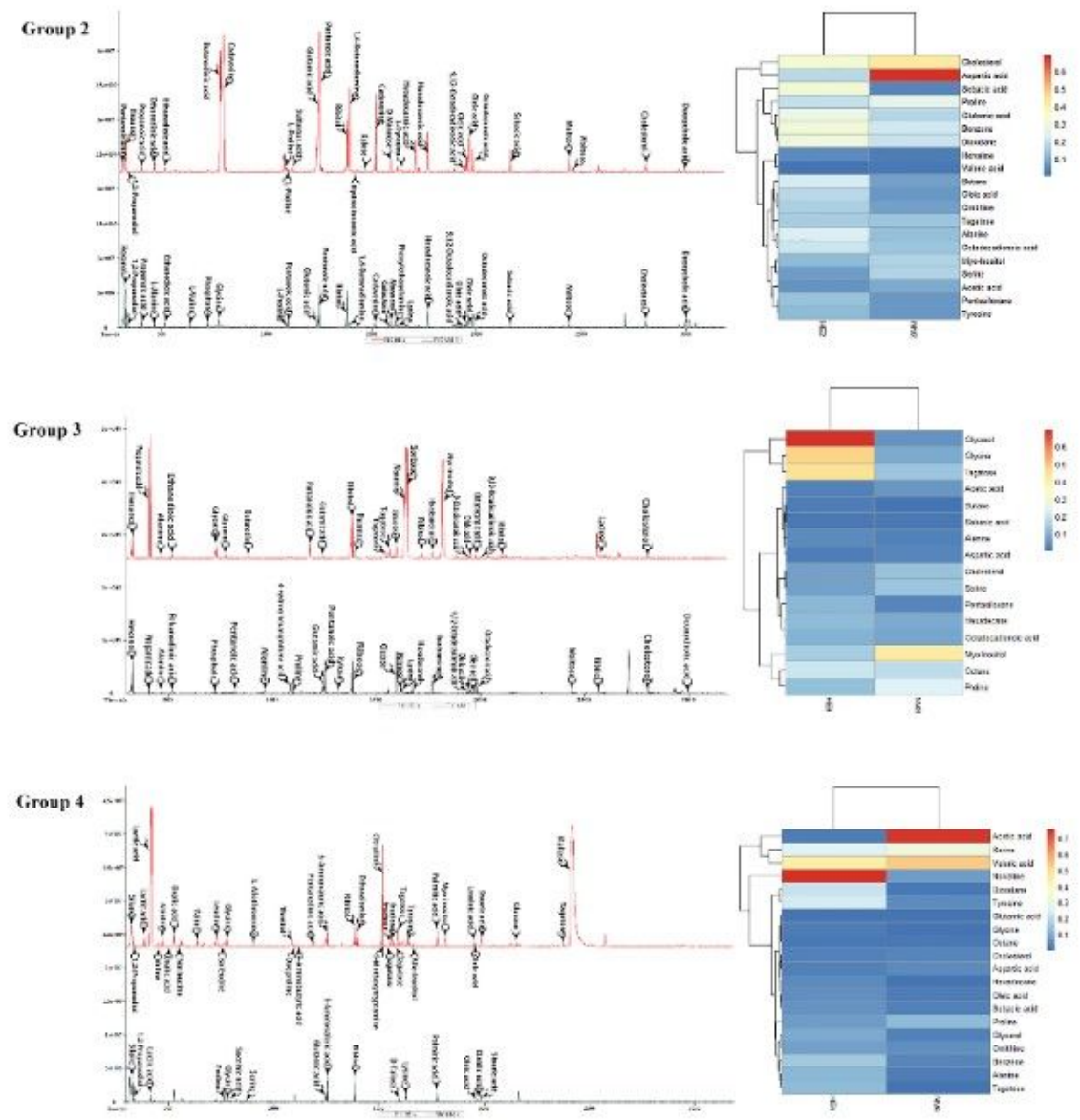

\section{Figure 3}

Peak value (A) and color-coded heat-map (B) analysis of CHE-related differential metabolites in group 1, group 2, group 3, and group 4. Hierarchical clustering of the metabolic profiling for HE patients and the normal in each group. The color scale represented the scaled abundance of each metabolite, denoted as Z-score, with red and blue indicating increased and decreased concentrations, respectively. 


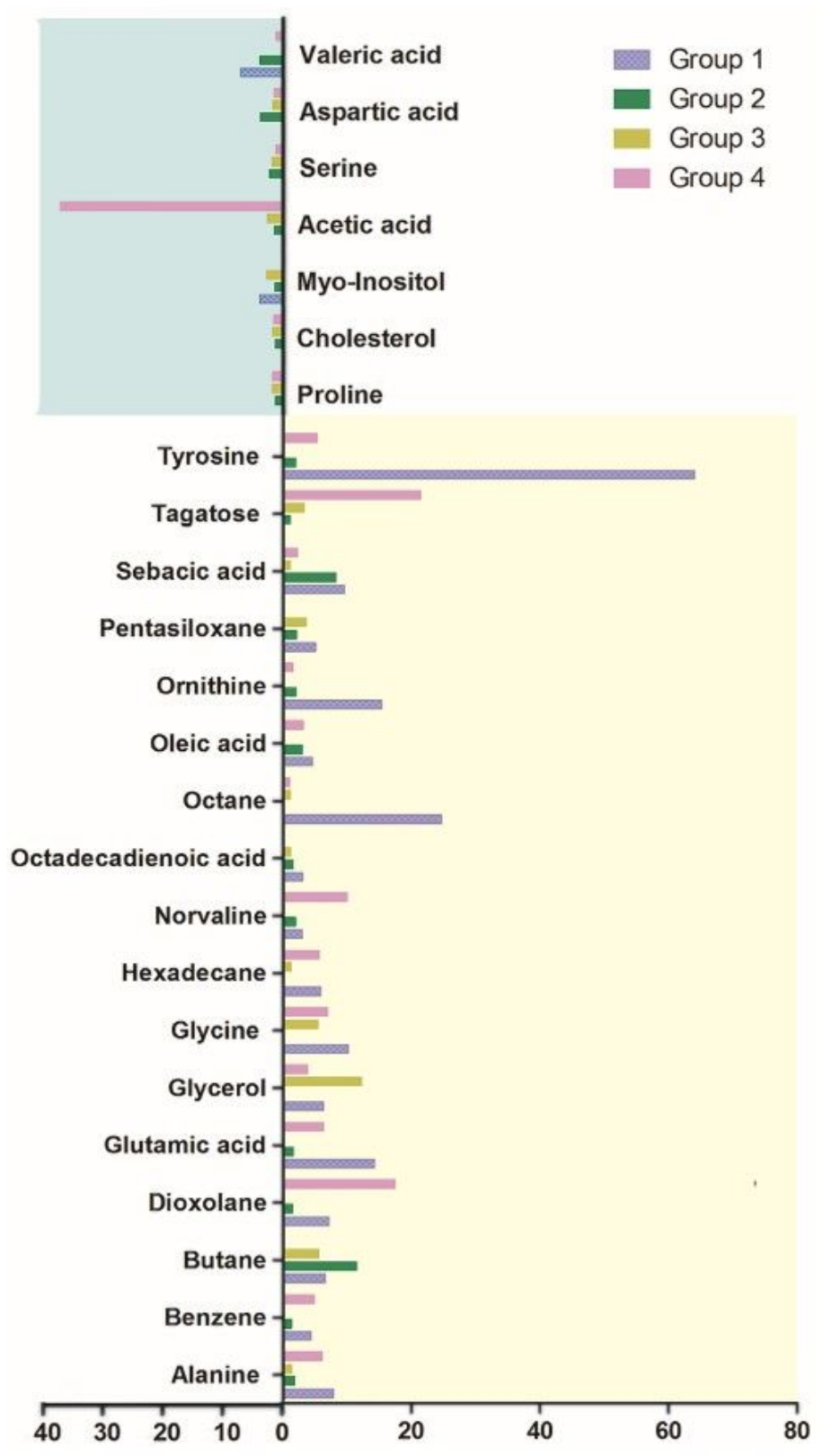

\section{Figure 4}

Multiple of difference of related metabolites The green background area represented the reduced metabolites in the patients, the values represented the ratio of the peak value of the metabolite in the normal divided by the peak value in the patient's body. The yellow background area represented the elevated metabolites in the patients, the values represented the ratio of the peak value of the metabolites in the patient's body divided by the peak value in the normal. 


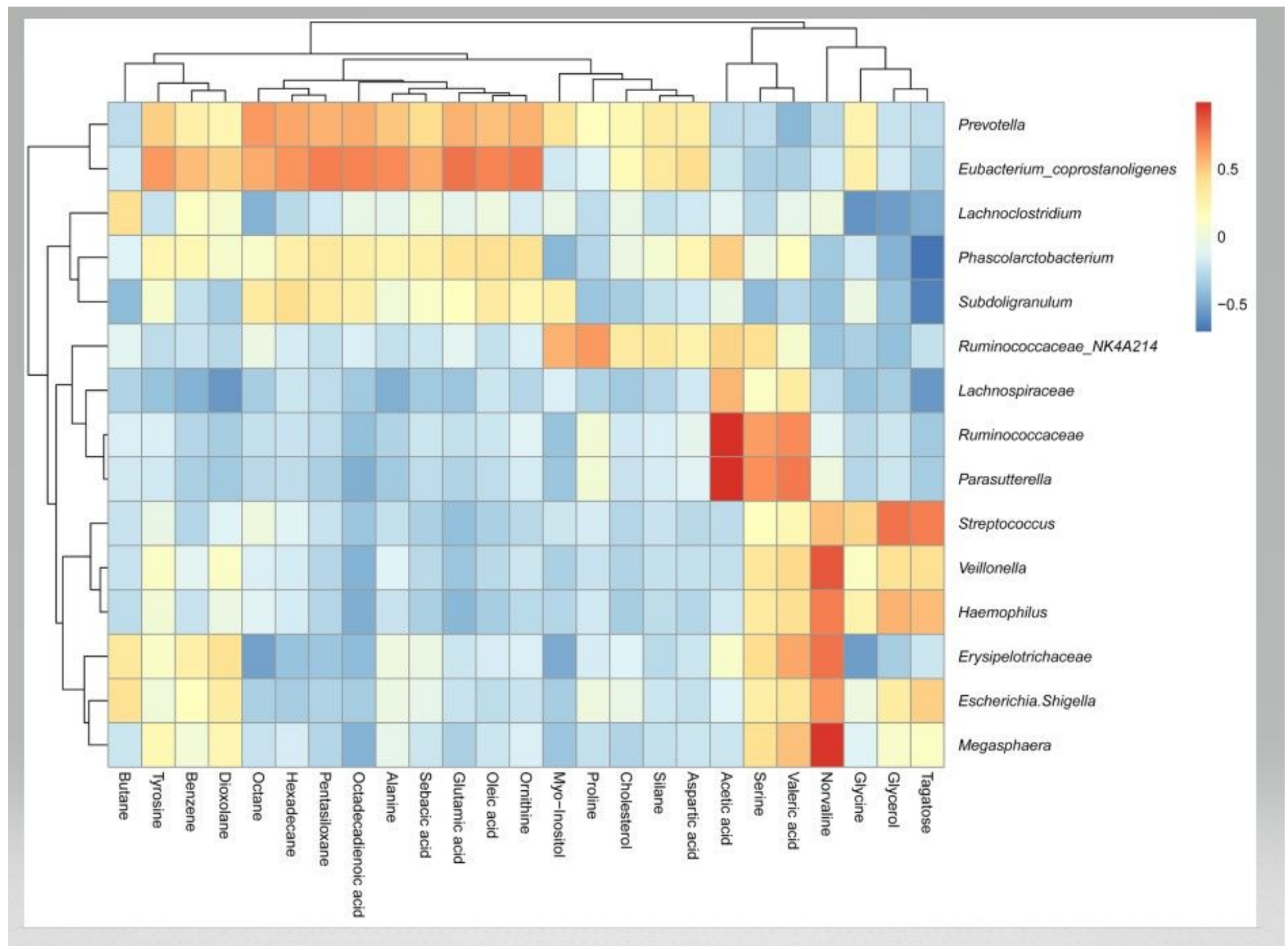

\section{Figure 5}

Correlation analysis between intestinal microbiota and metabolites differentiated in CHE patients compared with the normal The color scale represented the Spearman's rank correlation coefficient, with red and blue indicating positive and negative correlation, respectively.

\section{Supplementary Files}

This is a list of supplementary files associated with this preprint. Click to download.

- Tables1.docx

- Tables2.docx 